\title{
Research on the control system of an amphibious transmission line Inspection UAV based on fuzzy PID control
}

\author{
Lu Shengbiao ${ }^{1}$, Xia Liangbiao ${ }^{1}$, Shi ying ${ }^{1}$, Han Xiping ${ }^{1}$, Pang Tong ${ }^{1}$, Cai Ruyi $^{2}$ \\ ${ }^{1}$ Guangxi power grid limited liability company, Yulin Power Supply Bureau, Yulin, China \\ ${ }^{2}$ School of Electrical Engineering, Wuhan University, Wuhan, China
}

\begin{abstract}
The amphibious UAV combines the advantages of multi rotor UAV and line inspection robot. It can carry out two working modes: air flight and online driving. It can observe the running state of transmission line and improve the quality of patrol. In this paper, a fuzzy PID control algorithm is proposed to make the amphibious UAV flight control more flexible and close to the transmission line, and the simulation and software control process design are carried out.
\end{abstract}

\section{Introduction}

UAV is an advanced unmanned self propelled vehicle, which has the ability of high altitude, long distance, fast operation and self operation. It can quickly patrol the transmission line and improve the efficiency of transmission line inspection.At present,UAV has a problem of insufficient endurance, limited vision, easy to defect, and higher quality of control personnel.The inspection robot needs to be manually installed on the conductor, which is heavy in weight and difficult to hoist. At the same time, the robot is slow in walking speed, difficult to surmount obstacles, and has long navigation time and poor practicability.

In this paper, a new design scheme of amphibious patrol unmanned air vehicle is proposed based on the advantages of the inspection robot and the multi-rotor UAV. The fuzzy PID control algorithm is used to make the flight control more flexible and close to the target.

\section{Method}

\subsection{Amphibious UAV inspection process}

The amphibious patrol UAV is improved in the basic structure of the rotorcraft, including the suspension rod, the sliding power device and so on. The amphibious UAV can be hung on the ground line for inspection. The other power unit is used to drive the $U$ theory. The UAV's obstacle crossing operation is completed by the flight unit and the patrol equipment[1].

Amphibious type UAV in flight line below the starting unit, the motor drives the propeller to rotate the rotor lift, flying close to the power line to guide hanging on the line, when the sensor determines has hanging off the motor, each rotor, and start line patrol unit. When the UAV meets the obstacle, it stops moving on the line and starts the flight unit again for the flight winding barrier[2].

\subsection{Basic action of amphibious UAV}

The amphibious UAV takes the multi rotor unmanned aerial vehicle as the foundation of the basic flight unit. The flight unit controls the change of lift by adjusting the speed of the propeller with the motor, so as to control the attitude and position of the aircraft.

The lift force produced by the propeller is proportional to the square of the angular velocity of the propeller, that is, the speed of the motor is doubled and the tensile force increases to the original number of times. The flight attitude of the aircraft can be controlled by controlling the motor drive by a given PWM signal. When the rotation speed of each propeller is the same, the reverse torque produced by the two adjacent propellers counteracts each other, and the whole horizontal force is in the equilibrium state[3]. The speed of each propeller is increasing or decreasing, which can guarantee the vertical rise or decline of the aircraft unit. If the total lift of each propeller is greater than gravity, then the whole aircraft will steadily increase and decrease. Otherwise, the sum of the lift of the rotor is equal to the weight of the aircraft, and when the speed is zero, the vehicle will remain hovering[4].

Because the UAV controls the flight by adjusting the motor speed, it will cause the system not very stable, so an appropriate control method is needed to control the flight of the aircraft.

2.3 Flight control process of amphibious UAV 
The control of amphibious UAV adopts closed loop control. It compares the expected value with the actual measured value, and constantly corrections the actual measured values to achieve the numerical value we need. A high flying (for example, ignoring the influence of the air resistance and the external environment), take off from the ground, the plane will be at a certain acceleration to accelerate the rise; when the speed reaches a certain value and larger and the target height difference, the drone into the uniform rising stage; when the height of the UAV and close to the target range that movement into the deceleration phase[5]. The position of flight expiration stops. As shown in the figure, the flexible approach to the specified height hover is achieved by controlling the acceleration of the deceleration.

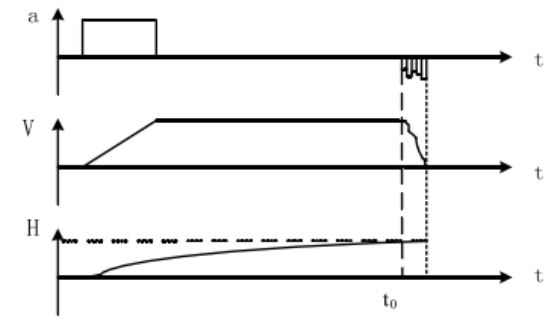

Fig. 1 Control deceleration acceleration method to fly to specified hover

The control process is adjusted by the difference between the actual height and the calibrated height as the adjustment. Considering the comprehensive factors, the fuzzy PID control principle is adopted for the handling of the control quantity. The general PID control can eliminate the static error, and can improve the control precision when the error value is relatively small. However, PID control has overshoot phenomenon, and at the same time, the reaction time is longer when there is interference. It is not suitable for direct flight control algorithm of amphibious UAV[6].

The fuzzy control has good robustness and can adapt to the change of parameters in a relatively large range. Compared with the traditional PID control, the dynamic response stability performance is high. The control of flight unit requires high real-time performance. Traditional PID can't meet the real-time requirement of controller[7]. Combining fuzzy control, it can shorten regulating time and meet real-time requirement, and also can restrain overshoot and overshoot behavior very well. Therefore, the combination of PID and fuzzy control can meet the design requirements.

\section{Research on fuzzy PID control algorithm}

\subsection{Fuzzy PID control}

Fuzzy PID control is a combination of fuzzy control and PID control. Fuzzy control is a nonlinear control. It is a numerical control based on fuzzy set theory, fuzzy language variable and fuzzy logic reasoning. It is the control method of fuzzy reasoning and decision processing of human behavior experience. Firstly, the control experience has been adapted into fuzzy rules, and then the real-time signal actually collected signal after fuzzification, fuzzy treatment as the input value input to the fuzzy inference module, output is obtained after the reasoning will be added to the controller, in order to achieve further control. The fuzzy controller is a kind of language controller, because the fuzzy control rules used are described according to the fuzzy condition statement in the fuzzy theory. The design contents of fuzzy controller include: first, select the set of all words that can describe the input and output variables, and then define the fuzzy subset of every fuzzy variable. Finally, we need to set up the corresponding fuzzy controller's control rule.

The change of the actual measurement error value and the calibration value of $\mathrm{E}$ and the error rate of $\mathrm{EC}$ as the input and processed by fuzzy controller (fuzzy parameters, fuzzy reasoning, fuzzy parameter solution) processing by the corresponding $\mathrm{Kp}, \mathrm{Ki}, \mathrm{Kd}$ values of three parameters input to the PID controller, the final output control the target object[8].

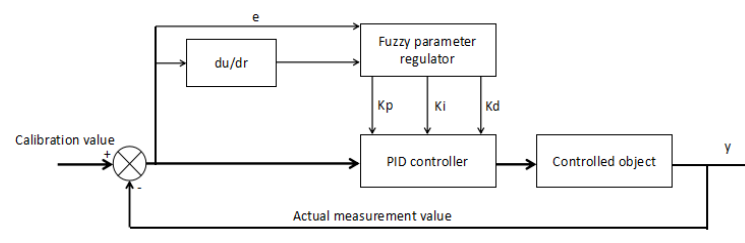

Fig. 2 control structure diagram

\subsection{Construction of PID fuzzy controller for amphibious UAV}

Fuzzy PID control is to find a fuzzy relationship based on PID, KD, KI, KP three parameters and $\mathrm{E}$ and $\mathrm{EC}$, and continuously modify all parameters in real time to meet the current control requirements of $\mathrm{E}$ and $\mathrm{EC}$, and build fuzzy PID controller under the condition of high control.

(1)Brushless DC motor transfer function

Assuming that the load of the brushless DC motor is constant, only the angular velocity of the output motor is considered, then the transfer function of the brushless DC motor is obtained.

$$
\frac{\Omega(s)}{U_{a}(s)}=\frac{K_{T}}{L J s^{2}+\left(L K_{D}+R J\right) s+K_{e} K_{T}+R K_{D}}
$$

Among them, $\mathrm{L}$ is self inductance of three phase stator, $\mathrm{R}$ is the resistance of stator windings in every phase, KT is expressed as electromagnetic torque constant, Ke back EMF constant, KD is the damping coefficient of rotation part, and $\mathrm{J}$ moment of inertia. According to the characteristics of the motor and the system, the values of all the parameters of the motor and the transfer function of the motor can be obtained.

$$
G(s)=\frac{523500}{s^{2}+87.35 s+10470}
$$

(2)Design of fuzzy PID controller

According to the existing control experience and the curve analysis of the measured value and the calibrated 
value, the adjustment is made. The size of the three parameters of the PID. Fuzzy control is used to improve the setting of three parameters of PID.

\subsection{System control simulation}

Based on the mathematical model of brushless motor, the traditional PID control and fuzzy PID control are simulated by Matlab/Simulink software, so as to verify the control effect of fuzzy PID.

After repeated experiments, the three proportional parameters of PID are set to $(0.4,1,0.01)$. The step signal sampling time is 0.001 , and the system running time is 2 seconds. The step response of the system can be obtained, as shown in Figure 3.

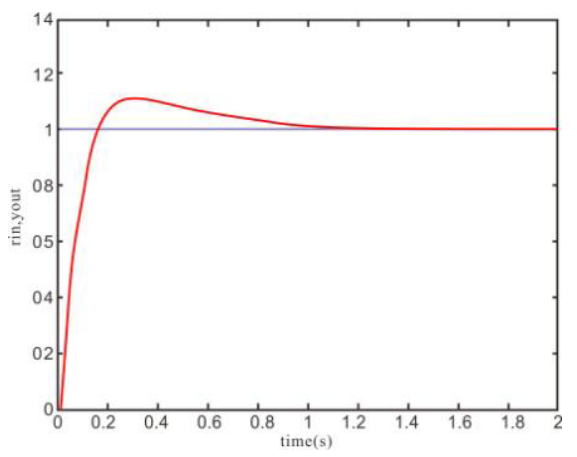

Fig. 3 Simulation results of step response

The simulation chart shows that the system can be adjusted to the specified position in a short time, but the phenomenon of overshooting occurs. This overshooting behavior is not allowed for the fly-flying patrol robot to fly to the specified height during flight. It is easy to collide with the transmission line.

Run the simulation program, the system step response shown in Figure 4. Under the step response, compared with the traditional PID in Fig. 3, the rise time and the adjustment time of the fuzzy PID control system are greatly reduced, the reduction of the overshoot is obvious, and the dynamic performance of the system is greatly reduced improve.

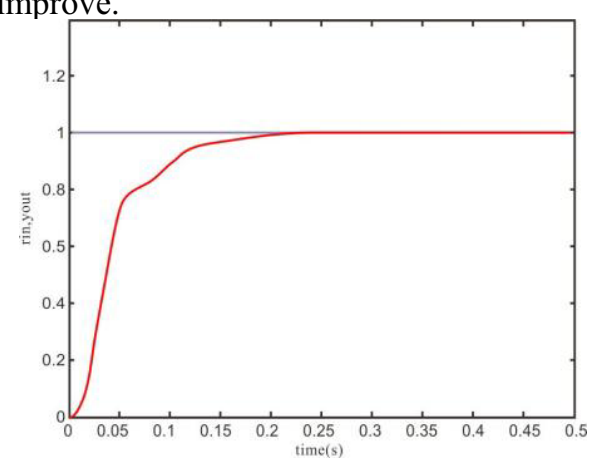

Fig. 4 Step response of fuzzy PID system

Based on this control algorithm, we also need to verify the system's response speed when the algorithm encounters interference. Therefore, at the position of the 400th sampling point of the algorithm, the amount of interference is added and the system responds to the system through Matlab, as shown in Figure 5.

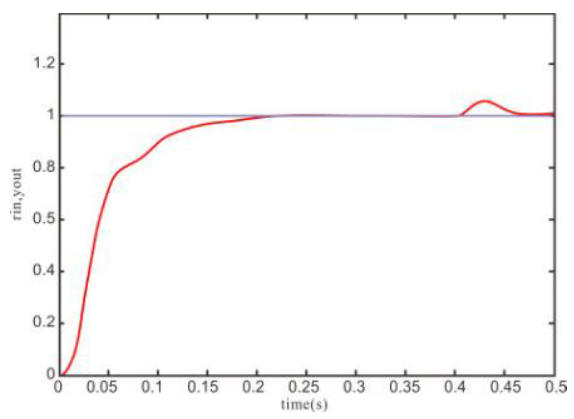

Fig. 5 Step response of system with interference

The simulation results show that overshoot occurs when $0.4 \mathrm{~s}$ is used, but the overshoot is relatively small, and at the same time, it can quickly and smoothly overshoot. Therefore, the flight control of the flying skid line robot can also respond to the system very quickly if it interferes[9].

The above simulation results show that fuzzy PID has good control effect, and fuzzy control algorithm is applied to the flight control of the flying skid line robot, which is in line with the design requirements.

\subsection{Design of flight control software}

The overall goal of the software design for the control system of the amphibious patrol UAV is to start the various functional modules of the flight control system and make it work normally, and achieve stable flight according to the established plan. The flight control of the UAV common and the biggest difference is that the amphibious patrol UAV flight in a transmission line for flight target, close to the target need to slow down and flexible contact, therefore, control of selecting the appropriate amount of control software design, using the control theory of fuzzy PID control algorithm to control the system. The main control chip of flight unit to complete the action process as follows: first, system initialization, and then check the flight system is normal, normal self start motor driver, the AD will open the collection and return attitude information, then execute the control algorithm to calculate the output control volume, will control the amount back to the motor driver.

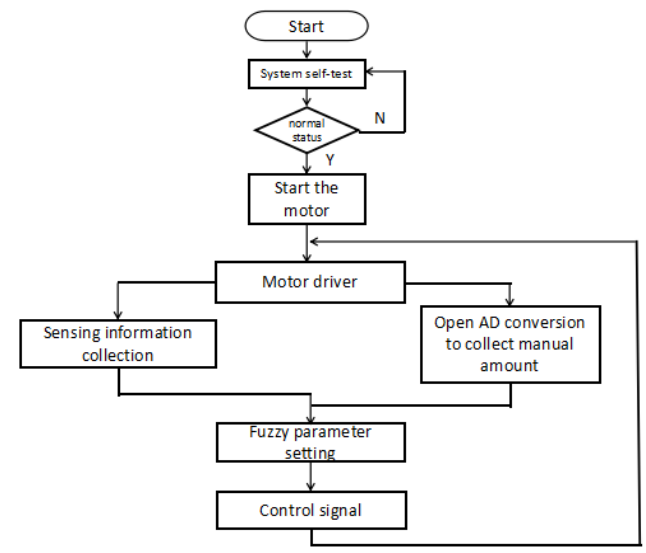

Fig. 6 system startup process 
In the process of navigation, the chip samples the result of the angular velocity and the linear acceleration in the result register of the ADC. The sampling flowchart is shown in Figure 7.

In motor control, first initialize hardware, setting control signal value is initialized; after the start, the attitude signal collected into reliable speed feedback signal according to the feedback signal by the fuzzy PID control algorithm parameter tuning output control signal, the motor reaches the desired speed, in order to adjust the attitude objective. The fuzzy PID controller software flow chart is shown in Figure 8.

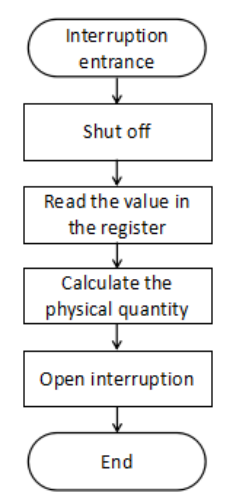

Fig. 7 sampling flow chart

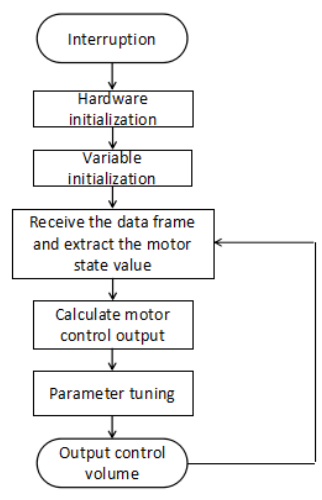

Fig. 8 software flow of fuzzy PID controller

\section{Conclusion}

The amphibious inspection UAV needs to fly near the transmission line and hangs on the transmission line. This paper designs the operation flow of the UAV flexible proximity transmission line, and designs and realizes the fuzzy PID control algorithm according to the operation flow. The simulation results show that the fuzzy PID control system can shorten the rise time and adjust the time, reduce the overshoot, and improve the dynamic performance of the system. However, it does not consider the impact of wind and other environmental factors. At the same time, the system intelligence is not high enough, Autonomous flight research.

\section{References}

1. Zheng Weigang. Research on distribution network inspection system base on infrared image technology for UAV[D]. Shenyang Agricultural University, (2013).

2. $\mathrm{Xu}$ Huadong. Research on Intelligent Obstacle Avoidance Methods for Power Lines Inspection with UAVs[D]. Nanjing University of Aeronautics and Astronautics, (2014).

3. Wu Feilong, Lin Han, Tang Mingwen. Application of Multi-relay Method in Inspection of Large Unmanned Aerial Vehicle Transmission Line[J]. Electric Power,02:104-110,(2015).

4. Liang Kun. Research on Ground Supervision and Control Technology of Power Line Inspection with Unmanned Aerial Vehicle[D]. Nanjing University of Aeronautics and Astronautics, (2014).

5. Whitworth C C,Duller A W G,Jones D I,et al. Aerial video inspection of overhead power lines [J].Power Engineering Journal ,15(1): 25-32, (2001).

6. Montambault S, Cote J, St.Louis,M.Preliminary results on the development of a teleoperated compact trolley for live-line working[C].Proceeding of the 2000 IEEE 9th International Conference on Transmission and Distribution Construction, Operation and Live-line Maintenance, Montreal, Canada:21-27(2000).

7. Wang Hongguang, Jiang Yong,Liu Aihua,et al.Research of Power Transmission Line Maintenance Robots in SIACAS[C]. 2010 1st International Conference on Applied Robotics for the Power Industry. Canada, October 5-7, (2010).

8. Jidai Wang,Aiqin Sun,Wei Su, et al.Development of an Expert Control Strategy for Controlling Obstacle Crossing of a High-voltage Transmission Line Inspection Robot[C]. Proceedings of the 18th International 195 Conference on Automation \& Computing, Loughborough University, Leicestershire,UK,8 September 2012.

9. Julien Beaudry, Kristopher Toussaint , Nicolas Pouliot. On the Application of VTOL UAVs to the Inspection of Power Utility Assets. International Conference on Applied Robotics for the Power Industry,(2010). 